\section{DIABETES-FRÜHERKENNUNG}

\section{Mitunter eine dermatologische Blickdiagnose}

- Bei 10-15\% aller Diabetiker erfolgt die erste Verdachtsdiagnose beim Hautarzt. Denn neun von zehn Diabetespatienten haben - bedingt durch Mikroangiopathie, Neuropathie und Immundefizienz - spezifische Hautprobleme, die anderen Krankheitsphänomenen oft lange vorangehen. Störungen der Hautbarriere plus die beeinträchtigte Immunabwehr prädestinieren sowohl Typ-1- als auch Typ-2-Diabetiker für bakterielle und mykologische Infektionen, so Prof. Marcus Maurer von der Berliner Charité. Wenn sich Diabetes-Risikokandidaten mit entsprechenden Hautbefunden vorstellen, sollte der Arzt ein Blutzuckertagesprofil veranlassen.

\section{Erythrasmen, Follikulitiden, Candidosen} Typisch sind Erythrasmen am Skrotum, in den Axillen oder den Oberschenkelinnenseiten, hervorgerufen durch chronische Infektionen mit Corynebakterien. Auch hartnäckige Follikulitiden oder ein Erysipel können Frühsymptom der Zuckerkrankheit

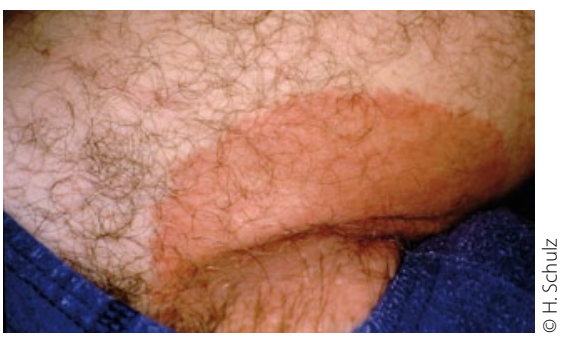

Diabetesindizien: Erythrasma an der Leiste ...

sein. Typisch, aber relativ unspezifisch sind Candida-Infektionen an Zehen und Nägeln.

\section{Kaum ein Diabetiker, den es nicht juckt} Eine weiteres häufiges Frühsymptom des Diabetes ist chronischer, oft therapieresistenter Juckreiz an Unterschenkeln und im Genitalbereich. Die Betroffenen sollten einmal wöchentlich die gesamte Hautoberfläche inkl. Fußsohlen inspizieren, möglichst mit einem Ganzkörperspiegel.

Ist der Diabetes bereits bekannt, kann eine optimale Blutzuckereinstellung die derma-

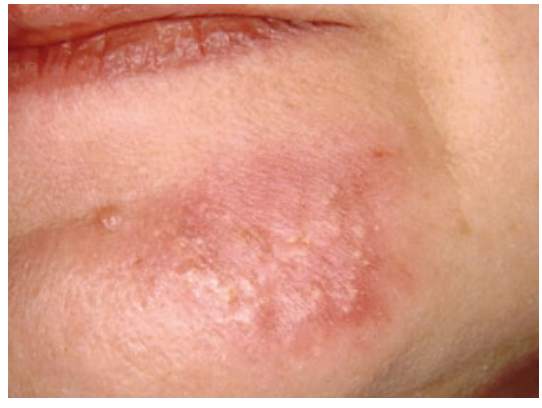

... und Follikulitis am Kinn.

tologischen Probleme verbessern. „Die Hautprobleme sind abhängig davon, wie gut der Diabetes eingestellt ist", so Maurer. Bei sehr trockener, schuppiger und rissiger Haut, eitrigen Hautveränderungen und Hautläsionen durch Schnitte oder Risse sollten Diabetiker zum Hautarzt überwiesen werden, rät Mauer. Prophylaktisch sollte nach der regelmäßigen Hautreinigung vor allem eine konsequente und ausreichende Rückfettung ohne sensibilisierende Substanzen erfolgen nach dem Grundsatz: Klotzen, nicht kleckern! DR. ANDREAS HÄCKEL .

Symposium "Von Kopf bis Fuß aufDiabetes eingestellt", DGIM-Jahrestagung 2012, Wiesbaden, April 2012 (unterstützt von Berlin Chemie)

\title{
Hepatitis C erhöht auch Sterberate durch nicht hepatische Ursachen
}

- Menschen mit chronischer Hepatitis $\mathrm{C}$ zu identifizieren und zu therapieren; lohnt sich. Denn bei ihnen ist nicht nur die hepatisch bedingte Sterberate erhöht. Eine chronische Infektion mit Hepatitis-C-Viren (HCV) erhöht auch die Sterberate durch extrahepatische Ursachen, und zwar um 35\%. Die Sterberate durch kreislaufbedingte Ursachen ist um $50 \%$ erhöht, ebenso jene für eine Reihe von Krebsarten - bis um das 8-Fache für Schilddrüsenkrebs.

Das hat eine Kohortenstudie aus Taiwan mit knapp 24000 Teilnehmern im Alter von 30-65 Jahren und einer Beobachtungszeit von gut 16 Jahren ergeben (J Inf Dis 2012; 206: 469). Die Gesamtsterberate war gegenüber jenen Teilneh- mern ohne chronische HCV-Infektion fast verdoppelt. Die hepatisch bedingte Sterberate lag um den Faktor 13 höher. Die erhöhten Sterberaten gelten dabei nur für Teilnehmer, bei denen HCV-RNA nachweisbar war, also tatsächlich eine chronische Hepatitis C vorlag. Waren nur HCV-Antikörper nachweisbar, gab es keine Unterschiede zu den Kontrollpersonen.

In einem Editorial zu dem Paper wird darauf hingewiesen, dass die Screeningstrategie von Risikogruppen, um die HCV-bedingte Sterberate in der Bevölkerung zu senken, versagt habe. Eine Ausweitung der Tests auf alle Menschen, die zwischen 1945 und 1965 geboren wurden, könne mehr Erfolg brin- gen. Eine solche Strategie wurde vor kurzem von der US-Seuchenbehörde CDC empfohlen.

Dass sich ein ausgeweitetes Screening auch in Deutschland lohnen könne, deutet eine Studie aus Berlin und Frankfurt am Main an. Hier wurde eine HCVSeroprävalenzrate von 1,6\% ermittelt das ist viermal so hoch wie bisherige Schätzungen (Plos One 2012; 7: e41206). Die Deutsche Leberhilfe e.V. setzt sich ebenfalls für vermehrte Tests ein. Da bei vielen Patienten mit VirusHepatitis der ALT-(GPT-)Wert erhöht ist, könnte ein solcher Test ein geeigneter Screeningparameter sein. Entsprechende Studiendaten werden für den Herbst erwartet.
RED : 